\title{
EDITORIAL
}

\section{A Systematic Review of Depression}

Depression is a widespread chronic medical illness that can affect thoughts, mood, and physical health. It is characterized by low mood, lack of energy, sadness, insomnia, and an inability to enjoy life. However, so far clinical studies have shown that patients with depression do not have a satisfactory therapeutic outcome. Therefore, based on recent studies, in this special issue these specialists will review depression systematically from behavior level to cellular and molecular level, and particularly focus on several interesting topics, such as "The neurobiological mechanisms and treatments of REM sleep disturbances in depression", "Research on the pathological mechanism and drug treatment mechanism of Depression", "The Antidepressant-like Effects of Estrogen-mediated Ghrelin", "The Effects of Calorie Restriction in Depression and Potential Mechanisms", "The effects of psychological stress on Depression" and "Herbal Medicine for Anxiety, Depression and

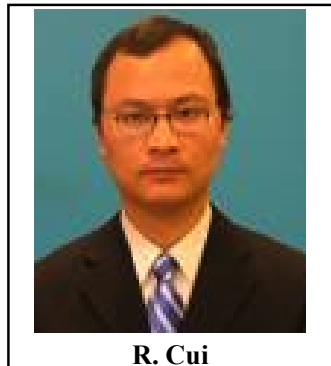
Insomnia". These reviews will provide new insight into treatment or adjunctive treatment for clinical depression.

Ranji Cui Ph.d \& Professo

(Guest Editor)

Second Hosipital Jilin University

Tel: 0086-0431-88796493

E-mails: cuiranji@jlu.edu.cn or cuiranji@hotmail.com 